\title{
Reducing power gradients in large-scale wind farms by optimal active power control
}

\author{
Simon De Rijcke \\ ESAT/ ELECTA \\ KU Leuven \\ Leuven, Belgium \\ Email: Simon.DeRijcke@esat.kuleuven.be
}

\author{
Johan Driesen \\ ESAT/ ELECTA \\ KU Leuven \\ Leuven, Belgium
}

\author{
Johan Meyers \\ Mechanics Department/ TME \\ KU Leuven \\ Leuven, Belgium
}

\begin{abstract}
Recently, new requirements have been imposed by grid regulators on power gradients or ramping rates of wind farms. These requirements are an important concern for wind farm operators, as they introduce additional losses in farm efficiency. In the current study, we investigate the use of optimal coordinated control of rotating kinetic energy reserves to smooth power gradients in a wind farm, while maximizing power extraction. To that end, we use turbulent velocity fields obtained from large eddy simulations of a large wind farm, and set up an elementary case in which we consider the coordinated control of two turbines only. We find that optimal control of kinetic energy reserves allows to significantly reduce power variations, keeping them within requirements on power gradients. Moreover, in comparison to a per-turbine limitation of gradients with a greedy power control, we extract on average $3 \%$ more power.
\end{abstract}

Index Terms-Wind power, optimal control, active power control, power gradient, turbulence.

\section{INTRODUCTION}

A growing number of incentives for the use of renewable energy sources has given the wind power market an incredible boost during the past decade. As a result farm owners have shifted their interest from small wind farms, equipped with small units, to larger offshore wind farms, with a turbine capacity up to several Megawatts. The average capacity of offshore wind farms grew from around $80 \mathrm{MW}$ in 2007 to $301 M W$ for farms currently under commission [1]. However, a larger share of wind power in the generation mix forces grid operators to rely on wind power to maintain a stable operating power system. As a result many European system operators start to impose limitations on ramping rates and power gradients in wind farms. In the current study, we investigate the use of optimal coordinated control of rotating kinetic energy reserves to smooth power gradients in a wind farm.

Changing weather patterns, wake effects, atmospheric turbulence, etc., create a time varying wind field throughout the farm, leading to large power variations and gradients. Moreover, in large wind-farms, self-induced turbulence levels

This work is sponsored by the Research Foundation - Flanders (FWO). The authors wish to acknowledge the financial support of the FWO. Project G.0376.12: Improving wind farm performance by active control of the atmospheric boundary layer using individual turbines as actuators. are much higher than usually encountered in the case of lone-standing turbines [2], further increasing the problem of power variability in modern farms. Such power variations may be reduced by temporarily depleting or increasing the rotating kinetic energy of the turbines (effectively changing their rotational speed) instead of directly converting fluctuations in velocity into electricity. Moreover, when considering the coordinated control of turbines on farm-level, a lot of additional opportunities arise for optimization, since local fluctuations at individual turbines may partly compensate each other.

Nowadays, most power stability studies that investigate the coupling of wind farms to the electricity grid, simplify the farm by an aggregated single-machine equivalent [3]-[5]. However, to optimally exploit internal farm variability for power smoothing, the dynamics of individual turbines need to be represented, and a realistic turbulent wind-flow pattern in wind farms is needed. To generate wind fields, different methods may be used, ranging from simple kinematic models to detailed numerical Large-Eddy Simulations (LESs) that generate realistic time-resolved turbulent flow solutions (cf. [6] for a review). In the current paper, we employ turbulent flow fields that are obtained from a large-eddy simulation study of a very large wind farm [7], [8], and use them to describe the dynamic wind fluctuations at individual turbines. We integrate this into an optimal control study in which we consider the coordinated control of two turbines in the farm, using their electrical torques (as function of time), to optimally control the rotating kinetic energy reserves of the turbines. We employ this approach to smooth power variations, and limit power gradients, keeping overall power output as high as possible.

This is further organized as follows. Section II motivates the approach and translates the objectives into an optimization problem. Next, section III applies the problem set-up to a two turbine case. First, results of reduced power variations with limited energy loss are shown. Secondly, a more in depth analysis of the precise behavior is discussed. Further discussion and conclusions are provided in section IV.

February 15, 2013 


\section{PRoblem SET-UP}

Current practice is to control wind farms by optimizing the power output of each wind turbine separately. According to this strategy, each turbine tracks a maximum power point. However, more onerous grid codes expect wind farms to be capable to accept and follow set points, for instance active and reactive power. Therefore, a central controller is a must [9]. Little is known about how this central controller decides upon which reference signals are send to each turbine. We tackle this question by optimization which decides about the contribution of each turbine.

\section{A. Objectives and constraints}

We consider the optimal control of a wind farm by means of the electrical torques $\boldsymbol{T}_{\boldsymbol{e}}(t)$ of all turbines in the farm. Here, $\boldsymbol{T}_{\boldsymbol{e}}$ is a vector containing the torques of individual turbines $T_{e, i}(t)$, with $i=1 \ldots N$ turbines. These torques dynamically depend on time, and are optimized in a time window $0<t<T$. We aim at two competing objectives, i.e. maximization of total power extraction $O_{1}$, and minimization of power variability $\mathrm{O}_{2}$, where

$$
\begin{aligned}
O_{1}\left(\boldsymbol{\omega}, \boldsymbol{T}_{\boldsymbol{e}}, T\right) & =\int_{0}^{T}-P_{f}(t)^{2} \mathrm{~d} t \\
& =\int_{0}^{T}\left(\sum_{i=1}^{N} T_{e, i} \omega_{i}\right)^{2} \mathrm{~d} t, \\
O_{2}\left(\boldsymbol{\omega}, \boldsymbol{T}_{\boldsymbol{e}}, T\right) & =\int_{0}^{T}\left(P_{f}(t)-P_{f}(t-\tau)\right)^{2} \mathrm{~d} t,
\end{aligned}
$$

with $P_{f}(t)$ the total farm power as function of time, and $\omega_{i}(t)$ the rotational speeds of individual turbines, and where we set $\tau=1 \mathrm{sec}$. We combine both objectives into one cost function, using a weighted sum, leading to following optimization problem:

$$
\min _{\boldsymbol{T}_{\boldsymbol{e}}}\left[w_{1} \cdot O_{1}\left(\boldsymbol{\omega}, \boldsymbol{T}_{\boldsymbol{e}}, T\right)+w_{2} \cdot O_{2}\left(\boldsymbol{\omega}, \boldsymbol{T}_{\boldsymbol{e}}, T\right)\right],
$$

where $w_{1}$, and $w_{2}$ are weights that need to be further selected (section III).

The optimization problem set-up in equation (3) is subject to turbine constraints. First, limits are set to all $i=1 \ldots N$ state variables, i.e. the rotational speeds $\boldsymbol{\omega}(t)$, and control variables $\boldsymbol{T}_{\boldsymbol{e}}(t)$ :

$$
\begin{aligned}
\omega_{\min } & \leq \omega_{i}(t) \leq \omega_{\max } \\
0 & \leq T_{e, i}(t)
\end{aligned}
$$

Although braking torques are sometimes used in emergency situations, the algorithm bounds all torques to positive values to avoid excessive fatigue. The pitch angles $\boldsymbol{\beta}$ remain constant because only the optimum speed operating region is considered (cf. discussion below). This is conform active power controllers [10]. In addition to the bounds, each turbine is subject to his motion equation, which is defined by the following ordinary differential equation

$$
J \frac{d \omega_{i}}{d t}=\frac{\kappa C_{p}\left(\lambda_{i}, \beta_{i}\right) u_{i}^{3}}{\omega_{i}}-T_{e, i},
$$

with $J$ the inertia constant, $\kappa$ a constant, $C_{p}$ the coefficient of performance and $u_{i}(t)$ the wind speed. The constant $\kappa=\rho A / 2$ is defined by the air density $\rho$ and the wind turbine rotor area $A=\pi R^{2}$, with $R$ the rotor radius. The aerodynamic coefficient of performance $C_{p}$ is modelled by [11], [12]

$$
\begin{aligned}
C_{p}\left(\lambda_{i}, \beta_{i}\right) & =c_{1}\left(c_{2} \gamma_{i}+c_{3} \beta_{i}+c_{4}\right) e^{c_{5} \gamma_{i}}, \\
\text { with } \quad \gamma_{i} & =\frac{1}{\lambda_{i}+c_{6} \beta_{i}}+\frac{c_{7}}{\beta_{i}^{3}+1},
\end{aligned}
$$

where $\lambda_{i}(t)=R \omega_{i} / u_{i}$ is the tip-speed ratio. The wind speed series $u_{i}(t)$ at the individual turbines are derived from turbulent flow fields created by a large-eddy simulation study. Hereby, a very large wind farm is simulated, taking into account the impact of increased turbulence [8]. For this study, only wind speed values between cut-in and rated wind speed, i.e. the optimum speed operating region, are considered.

The optimization problem stated in (1)-(6) is solved by a gradient based solver from Tomlab/Knitro [13]. The adjoint method is most suited to calculate the gradient of a scalar function with respect to a high number of parameters [14]. Contrary to the forward method or using finite differences, this method's computational effort is relatively insensitive to the number of parameters. With the aim to use the gradient calculation as input for a gradient based solver, this argument is highly relevant.

The optimization is performed over a predefined time period. For this period, a perfect forecast is assumed and the wind velocity field is not affected by wind farm control. This benchmark case gives the best achievable result given the assumptions made. However, it provides insight into the coordination of two turbines to achieve a smooth wind farm power output without hampering the maximum energy yield significantly.

\section{SMOOTHING POWER VARIATIONS: RESULTS FOR A TWO TURBINE CASE}

This section proves the concept of optimizing the wind farm power for a two turbine case. Both turbines are located in a large wind farm, including all turbulence which originates in such a farm (section II). The gradient based solver is used to run a time period $T=10 \mathrm{~min}$. The weighted sum of objective functions $O_{1}$ and $O_{2}$ introduced in section II determines the outcome in Fig. 1. First, the optimization runs for a weight combination of $w_{1}=1$ and $w_{2}=0$. Thus, the emphasis is only on maximizing the energy yield. The resulting solid curves in Fig. 1 show the normalized active power of each turbine and the wind farm. Notice that a some smoothing is already achieved by aggregating both turbines. Secondly, a weight combination of $w_{1}=0.5$ and $w_{2}=0.5$ is used, putting equal emphasis on $O_{1}$ and $O_{2}$. The resulting power output is depicted by dashed curves in Fig. 1 .

In accordance with the weights, active power variations are smoothed from solid to dashed curves. The curves show an overall smoother power output for both turbines separately, 


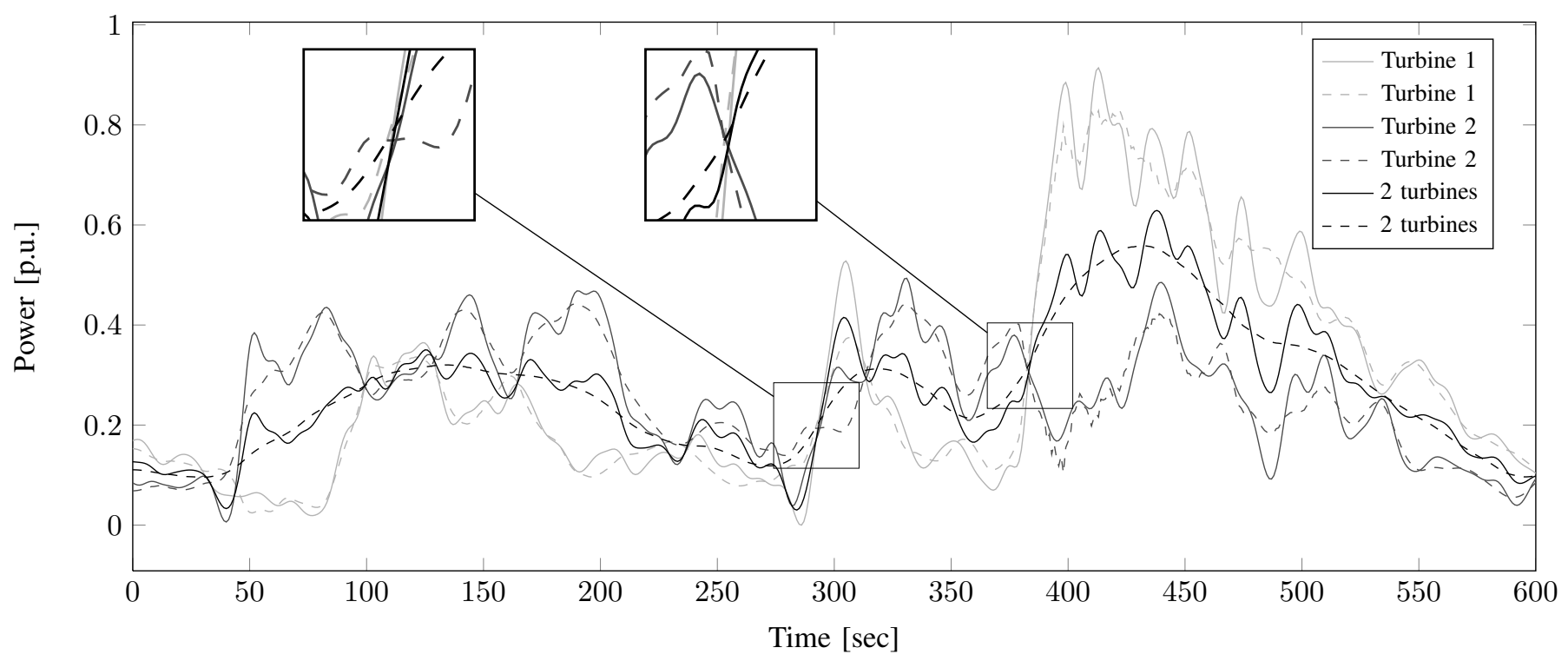

Fig. 1: Active power output for two turbines after optimizing the energy yield (solid curve, $w_{1}=1$ and $w_{2}=0$ ) and optimizing the energy yield in combination with a reduction in power variations (dashed curve, $w_{1}=0.5$ and $w_{2}=0.5$ ).

which reduces fatigue. As expected, the result is most striking for the wind farm power. At first sight, this outcome arises from the reduction of high frequency variations. However, the mitigation of power variations is not only due to eliminating high frequency fluctuations for both turbines separately. There is a trend in turbines cooperating to achieve the predefined objective on the farm level. The first close-up in Fig. 1 shows an upward power gradient for each turbine and consequently also the farm. To reduce the farm power gradient, both turbines contribute and reduce their power increase. The second closeup demonstrates this cooperation even better. To reduce the ramp-up, Turbine 1 evidently also reduces the power increase. In contrast, Turbine 2 decreases the active power faster. Also notice that respectively all solid and dashed curves cross each other because power is normalized with respect to the capacity of either one or two turbines.

These observations motivate to send distinct signals to each turbine. These signals are based on the status of each turbine with respect to the wind farm power, represented in the overall objective. If not, optimizing power variations of each turbine separately would result in higher variations.

\section{A. Limiting the ramping rate}

The variable nature of wind transforms farms into fluctuating power plants which are difficult to control. This makes frequency control a more difficult job, especially for isolated power systems with a lack of interconnections to neighbouring systems or generation mixes with a low flexibility. As a result, the responsible system operators started to impose limits on power gradients of wind farms [15], [16]. A trend which will likely spread to other power systems with higher instantaneous shares. Mostly, enforcing a limited power gradient is perceived to directly cut the available power, which is depicted in Fig.
2 [17]. Consequently, these necessary capabilities have led to much controversy because they lower the energy yield and thus the income of wind farm owners.

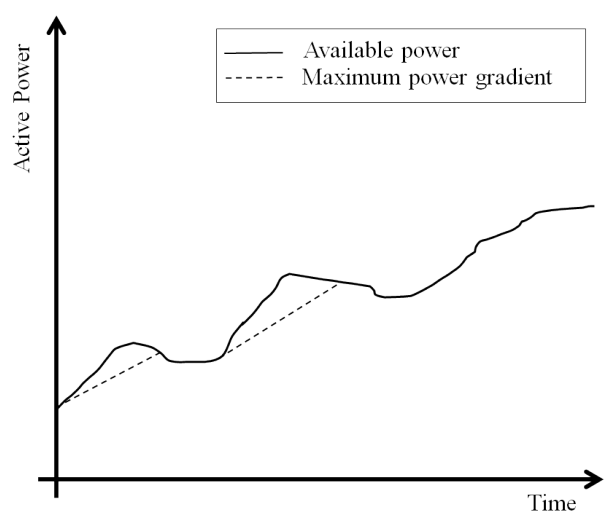

Fig. 2: Typical power gradient limit.

The conventional and most straightforward approach sets the power increase to a maximum value once the power gradient exceeds a limit. The dotted curve in Fig. 3 shows the resulting power with respect to the available power (solid curve) for the same two-turbine case as above. Only upward power gradient reductions are considered. The same limit on power gradients is achieved by the optimization algorithm. Therefore, the objective functions to maximize the energy yield and minimize the power gradient are combined by choosing the proper weights $w_{1}=0.5$ and $w_{2}=0.5$. The result, as a dashed curve, is also depicted on this Fig. 3.

The optimization algorithm succeeds to reduce the energy loss with $3 \%$. While the conventional method ends up with an energy loss of $3.9 \%$ relative to the available power, this 


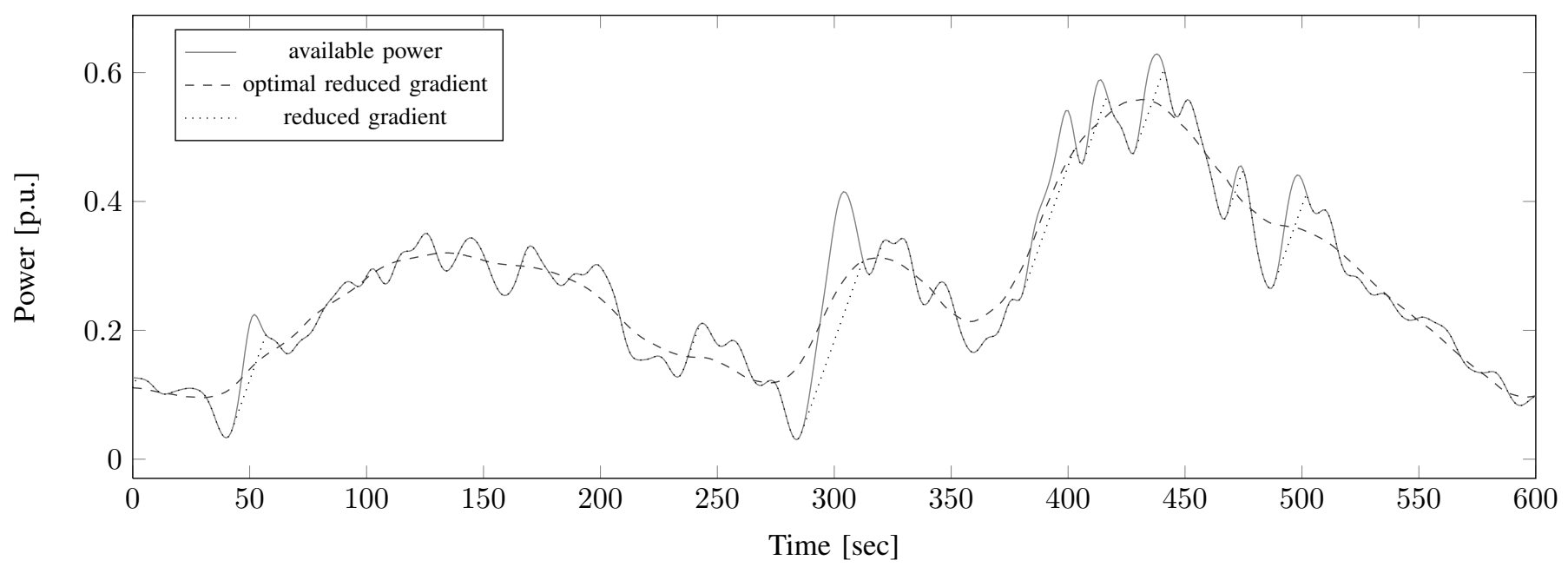

Fig. 3: Active power output for two turbines after optimizing the energy yield (solid curve) and optimizing the energy yield in combination with a reduction in power variations (dashed curve). The conventional approach to limit power variations is depicted by the dotted curve, mostly coinciding with the solid curve unless the gradient is limited.

energy loss is reduced to $0.9 \%$. A number that can decrease if only upward power variations are penalized. In addition, the result is a much smoother power output, also for negative power gradients. The maximum power gradient is reached only once, compared to several times for the conventional method.

\section{B. Reflection on the turbine behavior}

This section provides a more detailed insight into the optimization problem by calculating the gradient of the objective function with respect to the control variables. As explained in section II, this gradient is calculated via the adjoint method and used by the solver to decide upon the next step. Although the behaviour discussed below tends to repeat itself, the analysis in this section is for one specific case. However, it indicates clearly that the behaviour of each turbine, with respect to an objective on the farm level, highly depends on the circumstances.

For this analysis, again two optimization runs are compared: one with $O_{1}$ as objective (1) and the second with a combination of $O_{1}$ and $O_{2}$ as objective (2). A snapshot of 25 seconds is used to study the behaviour. Figs. $4 \mathrm{~b}$ and $4 \mathrm{a}$ respectively show the gradient of objective function $O_{1}$ and $\mathrm{O}_{2}$, for the result from optimization run (1). These graphs show the change of respectively the energy yield and the squared sum of all variations with respect to a positive change in torque for each time step. The graph in Fig. 5a shows the difference in torque between optimization run (1) and (2), or $\Delta T_{e, i}=T_{e, i}^{(2)}-T_{e, i}^{(1)} / T_{e, \max }$. Thus, a positive value reflects a higher torque and vice versa. Graph $5 \mathrm{~b}$ shows the power output for run (1) and (2).

To limit the upward wind farm ramp in Fig. 5b, one might suggest to reduce the upward ramp of Turbine 2. Therefore, the torque is increased between 35 and $45 \mathrm{sec}$, which is also according to both gradients in Fig. 4. They indicate a reduction in the objective functions due to an increase in the torque. Thereafter, the torque is reduced. In addition, Turbine 1 tends to cooperate. Although the power output is quasi constant, a downward rate is induced to reduce the farm power gradient. The slightly negative gradients, advising an increase of the torque, trigger this action.

The actions described are sometimes difficult to track, especially for smaller variations because priority is to reduce the highest power gradients. Moreover, this behaviour highly depends on the circumstances, i.e. the wind velocity field of the whole wind farm. A gradient study learns that much of this behaviour follows from anticipation on the incoming wind speed and coordination between the turbines. This anticipation highlights the importance of ongoing research about wind scanners. Such devices determine the ability of controllers to imitate this behaviour. Moreover, the necessary coordination motivates further research on farm controllers and dispatch.

\section{SUMMARY}

In this paper, we investigate the use of optimal coordinated control of kinetic energy reserves to smooth power variations in a wind farm, while maximizing the energy yield. To this end, an elementary case of two turbines is analysed thoroughly. These turbines are located in a large wind farm, including the dynamics of increased turbulence. We find that the optimal torque control significantly reduces power variations. In addition, we extract $3 \%$ more power compared to the conventional limitation of power gradients. Finally, a gradient analysis provides more insight into the coordination of electrical torque of each turbine. From this analysis, we observe that anticipation and coordination of turbines is a key element to achieve this energy reduction. 


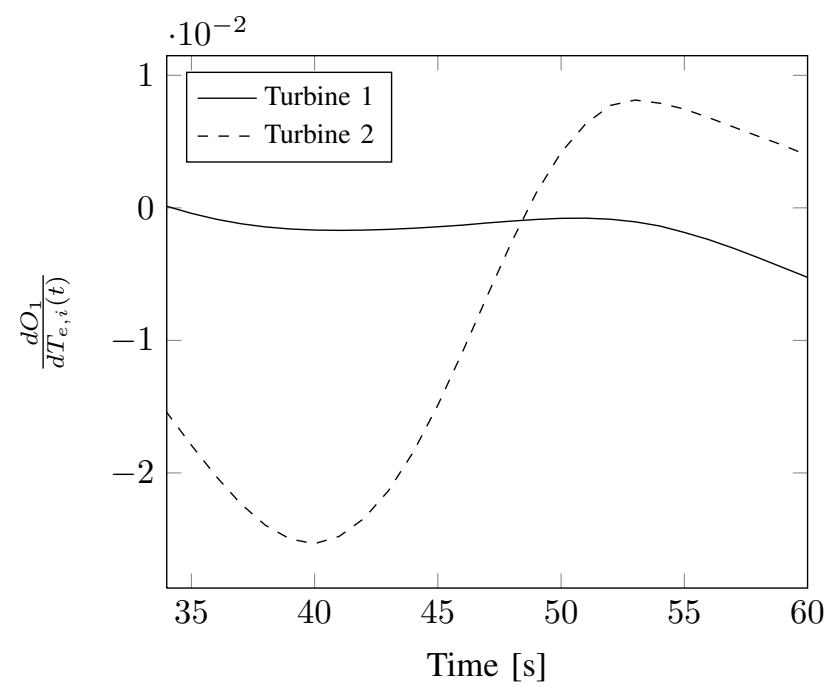

(a) $\frac{d O_{1}}{d T_{e, i}(t)}$

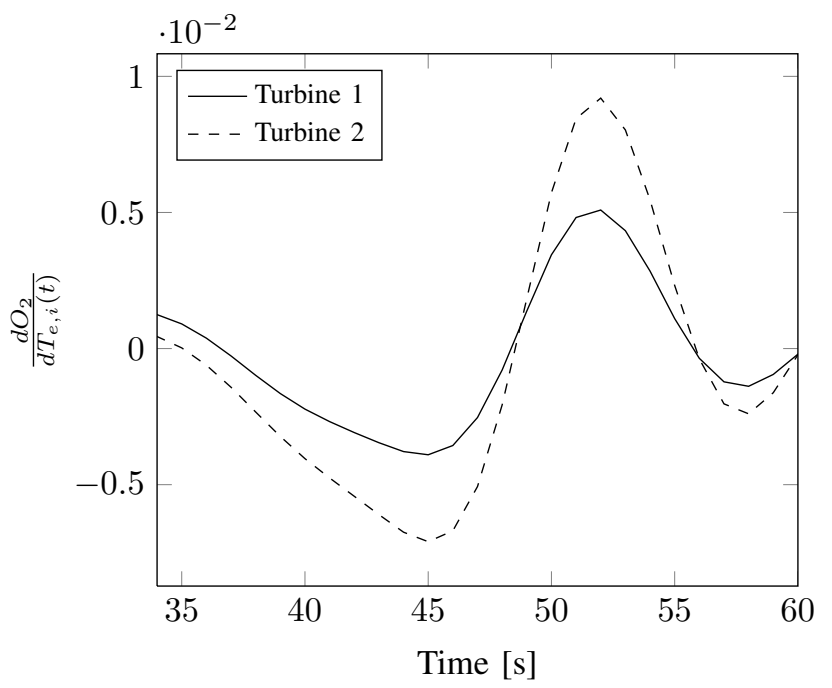

(b) $\frac{d O_{2}}{d T_{e, i}}$

Fig. 4: Snapshot of the gradient of objective functions $O_{1}$ and $O_{2}$ with respect to the torques of both turbine 1 and 2, defined at each time step.

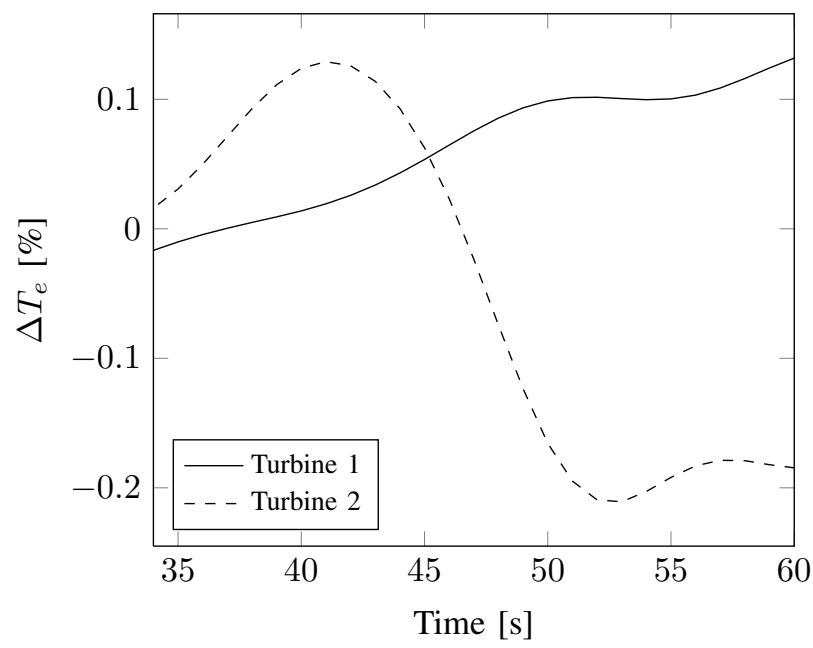

(a) Torque difference $\Delta T_{e}$

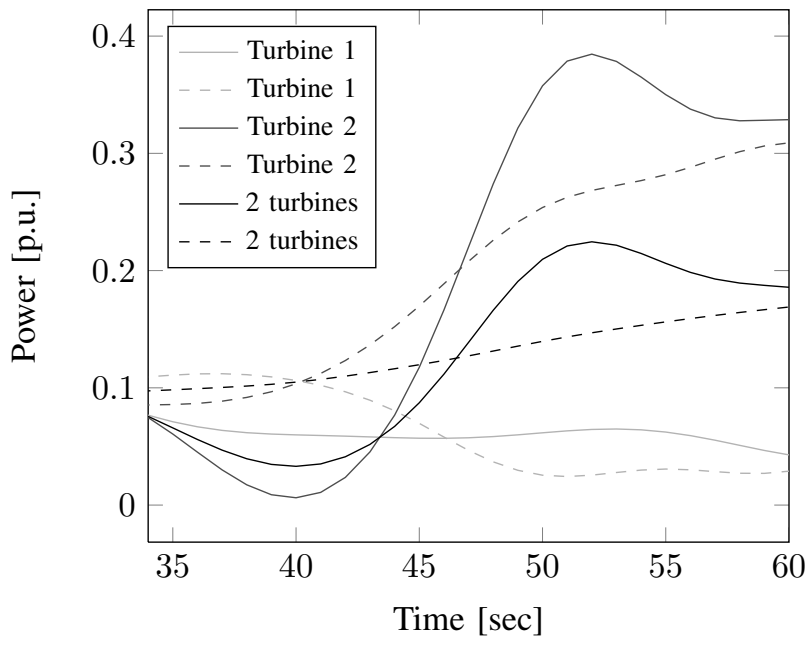

(b) Active power

Fig. 5: (a) Snapshot of the change in torque $\Delta T_{e}$ between the optimal trajectory of two optimization runs: one optimizing the energy yield and one optimizing the energy yield in combination with a reduction in power variations. (b) Active power output for two turbines after optimizing the energy yield (solid curve) and optimizing the energy yield in combination with a reduction in power variations (dashed curve).

\section{REFERENCES}

[1] European Wind Energy Association, "The european offshore wind industry - key trends and statistics 2011," EWEA, Tech. Rep., 2011.

[2] J. Lebron, C. L., and C. Meneveau, "Experimental study of the kinetic energy budget in a wind turbine stream-tube," Journal of Turbulence, vol. 13, no. 23, 2012.

[3] M. Pöller and S. Achilles, "Aggregated wind park models for analyzing power systems dynamics," in Fourth international workshop on largescale integration of wind power and transmission networks, October 2003, p. 10 pp.

[4] J. Conroy and R. Watson, "Aggregate modelling of wind farms containing full-converter wind turbine generators with permanent magnet synchronous machines: transient stability studies," IET Renewable power generation, vol. 3, no. 1, pp. 39-52, 2009.

[5] J. G. Slootweg and W. L. Kling, "Aggregated modelling of wind parks in power system dynamics simulations," in IEEE Bologna Power Tech Conference Proceedings, June 2003.

[6] A. Crespo, J. Hernández, and S. Frandsen, "Survey of modelling methods for wind turbine wakes and wind farms," Wind Energy, vol. 2, pp. 1-24, 1999.

[7] M. Calaf, C. Meneveau, and J. Meyers, "Large eddy simulations study of fully developed wind-turbine array boundary layers," Physics of fluids, vol. 22, 2010

[8] J. Meyers and C. Meneveau, "Optimal turbine spacing in fully developed 
wind farm boundary layers," Wind Energy, vol. 15, pp. 305-317, 2012.

[9] A. Hansen, P. Sorensen, F. Iov, and F. Blaabjerg, "Centralised power control of wind farm with doubly fed induction generators," Renewable Energy, vol. 31, pp. 935-951, 2006.

[10] A. Perdana, "Dynamic models of wind turbines," Ph.D. dissertation, Chalmers university of technology, 2008

[11] J. G. Slootweg, H. Polinder, and W. L. Kling, "Dynamic modelling of a wind turbine with doubly-fed induction generator," in Vancouver IEEE Power Engineering Society Summer Meeting, 15-19 July 2001.

[12] S. Heier, Grid integration of wind energy conversion systems, 2nd ed. John Wiley and Sons, 2006.

[13] P. Rutquist and M. Edvall, User's Manual for TOMLAB 1260 SE Bishop Blvd Ste E, Pullman, WA 99163, USA: Tomlab Optimization Inc., November 2008. [Online]. Available: http://tomopt.com/docs/TOMLAB.pdf

[14] Y. Cao, S. Li, L. Petzold, and R. Serban, "Adjoint sensitivity analysis for differential-algebraic equations: the adjoint dae ssytem and its numerical solution," SIAM journal on scientific computing, vol. 24, pp. 1076-1089, 2003.

[15] Eirgrid, "Eirgrid grid code version 3.3: Wind grid code only," Eirgrid, Tech. Rep., 2009

[16] Energinet, "Technical regulation 3.2.5 for wind power plants with a power output greater than 11 kw." Energinet, Tech. Rep., 2010

[17] M. Tsili and S. Papathanassiou, "A review of grid code technical requirements for wind farms," IET Renewable power generation, vol. 3, no. 3, pp. 308-332, 2009 